\title{
On the geodetic iteration number of a graph in which geodesic and monophonic convexities are equivalent
}

\author{
Marina Moscarini $(*)$ \\ (*) Department of Computer Science \\ Sapienza University of Rome, Italy
}

December 30, 2019

\begin{abstract}
Let $G$ be a graph, $u$ and $v$ two vertices of $G$, and $X$ a subset of $V(G)$. A $u-v$ geodesic is a path between $u$ and $v$ of minimum length. $I_{g}(u, v)$ is the set of vertices that lie on any $u-v$ geodesic and $I_{g}(X)$ is the set $\bigcup_{u, v \in X} I_{g}(u, v) . \quad X$ is $g$-convex if $I_{g}(X)=X$. Analogously, $I_{m}(u, v)$ is the set of vertices that lie on any induced path between $u$ and $v$ and $I_{m}(X)$ is the set $\bigcup_{u, v \in X} I_{m}(u, v) . X$ is $m$-convex if $I_{m}(X)=X$.

The $g$-convex hull $[X]_{g}$ of $X$ is the smallest $g$-convex set containing $X$. $I_{g}^{h}(X)$ equals $I_{g}(X)$, if $h=1$, and equals $\left.I_{g}^{h-1}(X)\right)$, if $h>1$. The geodetic iteration number, $\operatorname{gin}(X)$, of $X$ in $G$ is the smallest $h$ such that $I_{g}^{h}(X)=I_{g}^{h+1}(X)=[X]_{g}$. The geodetic iteration number of $G$, denoted by $\operatorname{gin}(G)$, is defined as $\operatorname{gin}(G)=\max \{\operatorname{gin}(X) \mid X \subseteq V(G)\}$.

In this paper we provide an $O\left(n^{3} m\right)$ time algorithm (where $n$ and $m$ are the cardinalities of the vertex set and of the edge set of the graph, respectively) to compute the geodetic iteration number of a graph belonging to the class, say $\Gamma$, of graphs in which the families of $g$-convex sets and of $m$-convex sets coincide (i.e., every $g$-convex set is $m$-convex). Since $\Gamma$ properly contains the class of distance-hereditary graphs, this result extends the result in [1]. Furthermore, we provide an $O\left(n^{2} m\right)$ time algorithm to compute the geodetic iteration number of a bipartite distance-hereditary graph.
\end{abstract}

Keywords convex hull, iteration number, geodesic convexity, monophonic convexity, distance-hereditary graphs, bipartite distance-hereditary graphs

\section{Introduction}

A convexity space on a connected graph $G$ is any set of subsets of $V(G)$ which contains the empty set, the singletons, and $V(G)$, and is closed under set in- 
tersection. Several graph convexity spaces have been defined using different path types; in particular, shortest paths (geodesics) and induced (or minimal or chordless) paths, were used to define geodesic convexity (or $g$-convexity) [2] [3] and monophonic convexity (or $m$-convexity) [2] [4], respectively. In [5] the class, say $\Gamma$, of graphs in which $g$-convexity and $m$-convexity are equivalent was introduced and characteristic properties of the graphs in $\Gamma$ (that allow to solve the problem of deciding the membership of a graph in $\Gamma$ in polynomial time) were stated. This class is a natural extension of the class of distance-hereditary graphs that are the graphs in which every induced path is a geodesic.

The geodetic iteration number of a graph, introduced in [6], is a measure of the "non $g$-convexity" of the family of the subsets of the vertex set of a graph.

In [1], the authors provide a characterization in terms of forbidden induced subgraphs of the distance-hereditary graphs whose geodetic iteration number is less or equal to a given positive integer. As a consequence of this result they provide an $O\left(n^{3} m\right)$ algorithm to compute the geodetic iteration number of a distance-hereditary graph.

In this paper, both an $O\left(n^{3} m\right)$ algorithm to compute the geodetic iteration number of a graph in $\Gamma$ and an $O\left(n^{2} m\right)$ algorithm to compute the geodetic iteration number of a bipartite distance-hereditary graph are provided.

The paper is organized as follows. After giving (Sections 2) basic graph theoretic definitions, we provide preliminary results concerning the separators and the prime components of a graph (Section 3) and relating the minimal vertex clique separators to the induced and shortest paths of a graph in the class $\Gamma$ (Section 4). In Section 5, we introduce the concept of "joint" of a set of vertices in a prime component and we prove that in a graph in $\Gamma$ the $g$-convex hull of a set $X$ of vertices is the union of the $g$-convex hulls of its joints. On the basis of this result, in Section 6, we state the main result concerning the computation of the geodetic iteration number of a graph in $\Gamma$. Finally, in Section 7 , we provide both an $O\left(n^{3} m\right)$ time algorithm to compute the geodetic iteration number of a graph in $\Gamma$ and an $O\left(n^{2} m\right)$ time algorithm to compute the geodetic iteration number of a bipartite distance-hereditary graph.

\section{Basic definitions}

In what follows, $G$ will be a finite, undirected, and simple graph. $V(G)$ and $E(G)$ denote the vertex set and the edge set of $G$, respectively. As usual we use the notation $u v$ for an edge $\{u, v\}$ and we denote by $n$ and $m$ the cardinalities of $V(G)$ and $E(G)$, respectively. $G$ is complete if every two distinct vertices of $G$ are adjacent.

In the following let $X$ be a nonempty subset of $V(G)$. The subgraph of $G$ induced by $X$, denoted by $G(X)$, is the graph $G^{\prime}$ such that $V\left(G^{\prime}\right)=X$ and $E\left(G^{\prime}\right)=\{e \in E(G) \mid e \subseteq X\} . X$ is a clique if $G(X)$ is complete. By $G-X$ $(G-v$ when $X=\{v\})$ we denote the induced subgraph $G(V(G) \backslash X)$. By $N(X)$ $(N(v)$ when $X=\{v\})$ we denote the neighbourhood of $X$ in $G$, i.e., the set of vertices in $V(G) \backslash X$ that are adjacent to a vertex in $X$. 
A path is a sequence $\left(v_{1}, \ldots, v_{k}\right), k \geq 1$, of distinct vertices of $G$ such that $v_{i} v_{i+1} \in E(G), 1 \leq i<k$. Let $p=\left(v_{1}, \ldots, v_{k}\right)$ be a path. $V(p)$ is the set of vertices appearing in $p$ and $E(p)$ is the set of edges consisting of a pair of vertices that are consecutive in $p$. The length $l(p)$ of $p$ is $|E(p)|=k-1$. The vertices $v_{1}$ and $v_{k}$ are connected and are called the endpoints of $p ; v_{i}$, $1<i<k$, is an internal vertex of $p$. The subsequence $\left(v_{i}, v_{i+1}, \ldots, v_{j}\right)$ of $p$, $1 \leq i \leq j \leq k$, is the $v_{i}-v_{j}$ subpath of $p$. A chord of $p$ is an edge $v_{i} v_{j} \in E(G)$, where $v_{i}$ and $v_{j}$ are not consecutive in $p$. A path is induced if it has no chords. If $p=\left(u=u_{1}, \ldots, u_{k}=v\right)$ is a $u-v$ path and $p^{\prime}=\left(v=v_{1}, \ldots, v_{h}=w\right)$ is a $v-w$ path such that $V(p) \cap V\left(p^{\prime}\right)=\{v\}$, then the concatenation $p p^{\prime}$ of $p$ and $p^{\prime}$ is the $u$ - $w$ path $\left(u=u_{1}, \ldots, u_{k}, v_{2}, \ldots, v_{h}=w\right)$.

A graph is connected if every two vertices are connected. The maximal connected induced subgraphs of a graph $G$ are its connected components. A connected graph $G$ is 2-connected if $G-v$ is connected, for each $v \in V(G)$. A graph is a block graph if every block (maximal 2-connected subgraph) is complete.

Henceforth, $G$ is a connected graph. Let $u$ and $v$ be two vertices of $G$ and $X$ a subset of $V(G)$. A $u-v$ geodesic is a $u-v$ path of minimum length. A graph $G$ is distance-hereditary if every induced path of $G$ is a geodesic.

The geodetic interval of $u$ and $v$, denoted by $I_{g}(u, v)$, is the set of vertices that lie on any $u-v$ geodesic and the geodetic interval of $X$, denoted by $I_{g}(X)$, is the set $\bigcup_{u, v \in X} I_{g}(u, v)$, with the convention that $I_{g}(\emptyset)=\emptyset . X$ is $g$-convex if $I_{g}(X)=X$. The $g$-convex hull $[X]_{g}$ of $X$ is the smallest $g$-convex set containing $X$. $I_{g}^{h}(X)$ is defined recursively as follows: $I_{g}^{0}(X)=X, I_{g}^{1}(X)=I_{g}(X)$, and $I_{g}^{h}(X)=I_{g}\left(I_{g}^{h-1}(X)\right)$ for $h>1$. The geodetic iteration number, $\operatorname{gin}(X)$, of $X$ in $G$ is the smallest integer $h$ such that $I_{g}^{h}(X)=I_{g}^{h+1}(X)$. The geodetic iteration number of $G$, denoted by $\operatorname{gin}(G)$, is defined as $\operatorname{gin}(G)=\max \{\operatorname{gin}(X) \mid X \subseteq$ $V(G)\}$.

The monophonic interval of $u$ and $v$, denoted by $I_{m}(u, v)$, is the set of vertices that lie on any induced $u-v$ path and the monophonic interval of $X$, denoted by $I_{m}(X)$, is the set $\bigcup_{u, v \in X} I_{m}(u, v)$, with the convention that $I_{m}(\emptyset)=$ $\emptyset$. $X$ is $m$-convex if $I_{m}(X)=X$. The $m$-convex hull $[X]_{m}$ of $X$ is the smallest $m$ convex set containing $X . I_{m}^{h}(X)$ is defined recursively as follows: $I_{m}^{0}(X)=X$, $I_{m}^{1}(X)=I_{m}(X)$, and $I_{m}^{h}(X)=I_{m}\left(I_{m}^{h-1}(X)\right)$ for $h>1$.

Fact 1. Let $G$ be a graph and $X$ a subset of $V(G)$.

1. $[X]_{g}=I_{g}^{h}(X)$, for every positive integer $h \geq \operatorname{gin}(X)$;

2. $[X]_{g}=\left[I_{g}(X)\right]_{g}$;

3. $[X]_{g} \subseteq[X]_{m}$.

We denote by $g(G)$ and $m(G)$ the family of the $g$-convex sets and the family of the $m$-convex sets of $G$, respectively.

Given a (finite) set $V$, a hypergraph $\mathcal{H}$ on the vertex set $V$ is a family of nonempty subsets of $V$ that covers $V$ (i.e., $V=\bigcup_{e \in \mathcal{H}} e$ ); the elements of $\mathcal{H}$ are the edges of $\mathcal{H}$. 


\section{$3 \quad$ Vertex separators and prime components}

In this section we state a number of results concerning the vertex separators and the prime components of a graph, which will be used in the following sections.

Let $G$ be a connected graph and $X \subseteq V(G)$. Let $K$ be a connected component of $G-X$ (observe that $N(V(K)) \subseteq X$ ); we denote by $\widehat{K}$ the subgraph of $G$ induced by $V(K) \cup N(V(K))$ and we say that $K$ is an $X$-component of $G$ if $N(V(K))=X$. Let $u$ and $v$ be two vertices of $G$; $u$ and $v$ are separated by $X$ if they belong to distinct connected components of $G-X ; X$ is a clique separator for $u$ and $v$ if $u$ and $v$ are separated by $X$, and $X$ is a clique; $X$ is a clique separator of $G$ if there exist two vertices for which $X$ is a clique separator; $X$ is a minimal separator for $u$ and $v$ if $u$ and $v$ are separated by $X$ and by no proper subset of $X . X$ is a minimal vertex separator of $G$ if there exist two vertices for which $X$ is a minimal separator. $X$ is a minimal vertex clique separator of $G$ if it is both a clique and a minimal vertex separator of $G$.

Fact 2. Let $G$ be a graph, $u$ and $v$ two vertices of $G$, and $X$ a subset of $V(G)$. $X$ separates $u$ and $v$ if and only if $X \cap\{u, v\}=\emptyset$ and, for every $u-v$ path $p$, $V(p) \cap X \neq \emptyset$.

Lemma 1. Let $G$ be a graph, $X$ a subset of $V(G)$, and $K$ a connected component of $G-X . N(V(K))$ separates every two vertices $u \in V(K)$ and $v \in V(G) \backslash$ $V(\widehat{K})$.

Proof. Let $u$ be a vertex in $V(K)$ and $v$ a vertex in $V(G) \backslash V(\widehat{K})$. Since no vertex in $V(K)$ is adjacent to a vertex in $V(G) \backslash V(\widehat{K})$, for every $u-v$ path $p$, $V(p) \cap N(V(K)) \neq \emptyset$ so that, by Fact $2, N(V(K))$ separates $u$ and $v$.

Lemma 2 ([5]). Let $G$ be a graph and $X$ a subset of $V(G) . X$ is a minimal separator for two vertices $u$ and $v$ if and only if $u$ and $v$ belong to two distinct $X$-components of $G$.

Lemma 3. Let $G$ be a graph, $X$ a clique of $G, K$ a connected component of $G-X$, and $u$ and $v$ two vertices in $V(G) \backslash V(K)$. One has that $I_{m}(u, v) \subseteq$ $V(G) \backslash V(K)$.

Proof. Suppose, by contradiction, that there exists an induced $u$ - $v$ path $p$ in $G$ such that $V(p) \cap V(K) \neq \emptyset$. Let $w \in V(p) \cap V(K)$. Let $p_{u w}$ be the $u$ - $w$ subpath of $p$ and $p_{w v}$ the $w-v$ subpath of $p$. Since $u$ is either in $X$ or in a connected component of $G-X$ distinct from $K$, by Fact $2, V\left(p_{u w}\right) \cap X \neq \emptyset$; analogously, $V\left(p_{w v}\right) \cap X \neq \emptyset$. Let $u^{\prime}$ be a vertex in $V\left(p_{u w}\right) \cap X$ and $v^{\prime}$ a vertex in $V\left(p_{w v}\right) \cap X$. Since $u^{\prime}$ and $v^{\prime}$ are distinct and not consecutive in $P$, and $\left\{u^{\prime}, v^{\prime}\right\} \subseteq V(p) \cap X$, $p$ is not induced (contradiction).

Corollary 4. Let $G$ be a graph, $X$ a clique of $G$, and $K$ a connected component of $G-X$. If $Y \subseteq V(G) \backslash V(K)$, then $[Y]_{m} \subseteq V(G) \backslash V(K)$.

Proof. By Lemma 3, one can easily prove, by induction, that, for every $j, j \geq 0$, $I_{m}^{j}(Y) \subseteq V(G) \backslash V(K)$. 
Two vertices are clique separable in $G$ if there exists a clique of $G$ separating them. A prime component of $G$ is a subgraph of $G$ induced by a maximal subset of $V(G)$ not containing two vertices that are clique separable in $G$. $G$ is prime if $G$ has only one prime component. The prime hypergraph $\mathcal{P}_{G}$ of a graph $G$ is the hypergraph whose vertex set is $V(G)$ and whose edges are the vertex sets of the prime components of $G$. Due to the maximality of a prime component, one has the following.

Fact 3. Let $G$ be a graph and $P$ a prime component of $G$. For every $u \notin V(P)$, there exists $v \in V(P)$ such that $u$ and $v$ are clique separable.

Lemma 5. Let $G$ be a graph, $P$ a prime component of $G$, and $K$ a connected component of $G-V(P)$. One has that $N(V(K))$ is a clique.

Proof. Suppose, by contradiction, that there exist two nonadjacent vertices $x$ and $y$ in $N(V(K))$. Since $x$ and $y$ are in $N(V(K))$ and $K$ is connected there exists an induced $x$-y path $p$ whose internal vertices are in $V(K)$. Let $u$ be an internal vertex of $p(u$ exists, since $x y \notin E(G))$. Since $u \notin V(P)$, by Fact 3, there exists a vertex $v \in V(P)$ and a clique $X$ such that $X$ separates $u$ and $v$. Since $p$ is induced, $X$ cannot contain two vertices not consecutive in $p$, so that $X$ does not separate $x$ and $u$ or $y$ and $u$. It follows that $X$ separates $x$ and $v$ or $y$ and $v$ (contradiction).

Lemma 6. Let $G$ be a graph, $P$ a prime component of $G$, and $K$ a connected component of $G-V(P)$. One has that $V(P) \backslash N(V(K)) \neq \emptyset$.

Proof. Suppose, by contradiction, that $V(P)=N(V(K))$ so that every vertex in $V(P)$ is adjacent to a vertex in $V(K)$ and, hence:

(a) for every pair of vertices $u \in V(K)$ and $v \in V(P)$, there exists an induced $u-v$ path having all vertices, except $v$, in $V(K)$.

Firstly, let us show that:

(b) given a nonempty subset $U$ of $V(P)$, if there exists a vertex $u \in V(K)$ such that $u$ is clique separable from no vertex in $U$ and is clique separable from a vertex $v \in V(P)$, then there exists a vertex $u^{\prime} \in V(K)$ that is clique separable from no vertex in $U \cup\{v\}$.

To this aim, let us show that

(c) every clique separating $u$ and $v$ must contain $U$.

Suppose, by contradiction, that there exist a clique $X$ separating $u$ and $v$, and a vertex $x \in U$ such that $x \notin X$. Since, by Lemma $5, V(P)$ is a clique, $x$ is adjacent to $v$ so that $X$ separates $u$ and $x$ (contradiction).

Let $p$ be an induced $u-v$ path such that $V(p) \backslash\{v\} \subseteq V(K)$ (such a path exists by $(a))$. By $(c)$ and Fact 2, every clique separating $u$ and $v$ must contain $U$ and at least one internal vertex of $p$. Let $u^{\prime}$ be the last vertex in (the sequence) $p$ belonging to a clique separating $u$ and $v$ (see Figure 1); then, by (c), $u^{\prime}$ is 


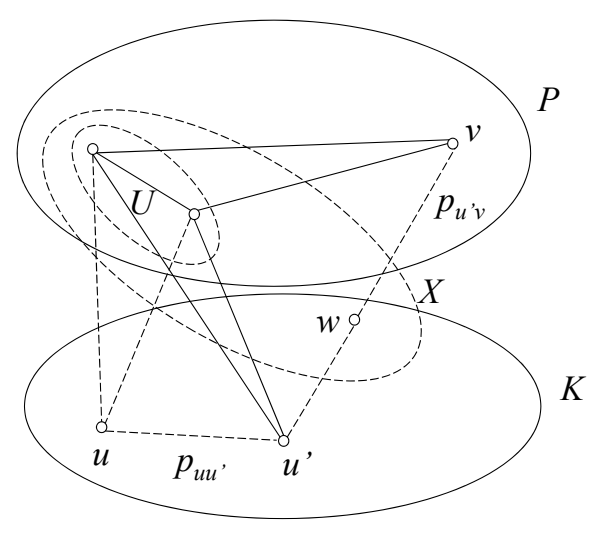

Figure 1:

adjacent to every vertex in $U$ and, hence, $u^{\prime}$ is clique separable from no vertex in $U$. In order to prove $(b)$, it remains to show that $u^{\prime}$ and $v$ are not clique separable. Suppose, by contradiction, that there exists a clique $X$ separating $u^{\prime}$ and $v$. Then, by $(c)$ and Fact $2, X$ contains $U$ and a vertex $w$ in the $u^{\prime}-v$ subpath $p_{u^{\prime} v}$ of $p$. Since $p$ is induced, $X$ cannot contain a vertex in the $u$ - $u^{\prime}$ subpath of $p$ so that, if $u$ and $v$ were connected in $G-X$, then $u^{\prime}$ and $v$ would be connected in $G-X$, contradicting the fact that $u^{\prime}$ and $v$ are separated by $X$. Therefore $X$ is a clique separating $u$ and $v$ and, since $w$ follows $u^{\prime}$ in (the sequence) $p$ a contradiction arises. Therefore, $u^{\prime}$ and $v$ are not clique separable and $(b)$ is proved.

Let $u_{1} \in V(K)$ be a vertex adjacent to a vertex in $V(P)$. Then $u_{1}$ is clique separable from no vertex in $N\left(u_{1}\right) \cap V(P)$; furthermore, by Fact 3, there exists a vertex $v_{1} \in V(P)$ such that $u_{1}$ and $v_{1}$ are clique separable. Therefore, by $(b)$, there exists a vertex $u_{2} \in V(K)$ such that $u_{2}$ is clique separable from no vertex in $N\left(u_{1}\right) \cap V(P) \cup\left\{v_{1}\right\}$. If $V(P)=N\left(u_{1}\right) \cap V(P) \cup\left\{v_{1}\right\}$, then, by Fact $3, P$ is not a prime component of $G$ (contradiction). Otherwise, there exists $v_{2} \in V(P)$ such that $u_{2}$ and $v_{2}$ are clique separable so that, by $(b)$, there exists $u_{3} \in V(K)$ such that $u_{3}$ is clique separable from no vertex in $N\left(u_{1}\right) \cap V(P) \cup\left\{v_{1}, v_{2}\right\}$, and so on. Therefore by applying $(b)$ a finite number of times, we reach a contradiction.

Theorem 7. Let $G$ be a graph, $P$ a prime component of $G$, and $K$ a connected component of $G-V(P)$. There exists a connected component $K^{\prime}$ of $G-N(V(K))$ containing $V(P) \backslash N(V(K))$; furthermore:

1. $K^{\prime}$ is an $N(V(K))$-component of $G$, and

2. $N(V(K))$ is a minimal clique separator for every two vertices $u \in V(K)$ and $v \in V\left(K^{\prime}\right)$ 
Proof. Since, by Lemma 6, $V(P) \backslash N(V(K)) \neq \emptyset$ and, by Lemma $5, N(V(K))$ is a clique, every two vertices in $V(P) \backslash N(V(K))$ are connected in $G-N(V(K))$ (otherwise $P$ would not be a prime component of $G$ ) and, hence, there exists a connected component $K^{\prime}$ of $G-N(V(K))$ such that $V(P) \backslash N(V(K)) \subseteq V\left(K^{\prime}\right)$. Proof of 1. Let us show that $K^{\prime}$ is an $N(V(K))$-component of $G$. Suppose, by contradiction, that there exists a vertex $w$ in $N(V(K)) \backslash N\left(V\left(K^{\prime}\right)\right.$ ) (observe that, since $K^{\prime}$ is a connected component of $\left.G-N(V(K)), w \notin V\left(K^{\prime}\right)\right)$ and let $w^{\prime}$ be a vertex in $V(P) \backslash N(V(K)) \subseteq V\left(K^{\prime}\right)$, By Lemma $5, N(V(K))$ is a clique and, hence, $N\left(V\left(K^{\prime}\right)\right)$ is a clique. Furthermore, by Lemma 1, $N\left(V\left(K^{\prime}\right)\right)$ separates $w$ and $w^{\prime}$. Since both $w$ and $w^{\prime}$ are in $\left.V(P)\right)$, a contradiction arises. Proof of 2. By Lemmas 5 and 2.

The next result relates prime components and convex hulls.

Theorem 8. Let $G$ be a graph, $X$ a subset of $V(G)$, and $P$ a prime component of $G$. If $X \subseteq V(P)$ then $[X]_{m} \subseteq V(P)$.

Proof. By Lemmas 5 and 3, one can easily prove, by induction, that, for every $j, j \geq 0, I_{m}^{j}(X) \subseteq V(P)$.

Since $\mathcal{P}_{G}$ is a cover of $V(G)$ the following holds.

Fact 4. Let $G$ be a graph and $X$ a subset of $V(G)$. One has that

$$
X=\bigcup_{V(P) \in \mathcal{P}_{G}} X \cap V(P) .
$$

Lemma 9 ([5]). Let $G$ be a graph. For every prime component $P$ of $G$, a nonempty subset $X$ of $V(P)$ belongs to $m(G)$ if and only if $X$ is a clique or $X=V(P)$.

Let $G$ be a graph, $X$ a subset of $V(G)$, and $P$ a prime component of $G$. In the following, $\mathcal{K}(X, P)$ is the set of the connected components $K$ of $G-V(P)$ such that $X \cap V(K) \neq \emptyset$.

Lemma 10. Let $G$ be a graph, $X$ a subset of $V(G)$, and $P$ a prime component of $G$. For every $j, j \geq 0, \mathcal{K}\left(I_{g}^{j}(X), P\right)=\mathcal{K}(X, P)$.

Proof. By induction.

Basis. $j=0$. Trivial.

Induction. $j>0$. By inductive hypothesis, $\mathcal{K}\left(I_{g}^{j-1}(X), P\right)=\mathcal{K}(X, P)$. Therefore $\mathcal{K}(X, P) \subseteq \mathcal{K}\left(I_{g}^{j}(X), P\right)$. Furthermore, if $K \notin K\left(I_{g}^{j}(X), P\right)$, then $I_{g}^{j}(X) \cap$ $V(K)=\emptyset$ and, hence, $X \cap V(K)=\emptyset$.

\section{The class $\Gamma$}

In this section, after recalling the characterization of the graphs in $\Gamma$ provided in [5], we state some results (which will be useful in the next sections) relating minimal vertex clique separators to induced paths and geodesics of a graph in the class $\Gamma$. 
Theorem $11([5])$. Let $G$ be a graph. $g(G)=m(G)$ if and only if

(1) $g(P)=m(P)$ for every prime component $P$ of $G$, and

(2) for every minimal vertex clique separator $S$ of $G$ and for every $S$-component $K$ of $G$ and for every vertex $u \in V(K) \cap N(S)$, the set $S \cup\{u\}$ is a clique.

In Figure 2 a graph in the class $\Gamma$ and its prime components $P_{1}, P_{2}, \ldots, P_{6}$ are shown.

Lemma 12. Let $G$ be a graph such that $g(G)=m(G)$ and $X$ a minimal vertex clique separator of $G$. For every induced path $p$ having an endpoint in an $X$ component of $G,|V(p) \cap X| \leq 1$.

Proof. Let $p=\left(u=w_{1}, w_{2}, \ldots, w_{k}=v\right)$ be an induced $u-v$ path such that $u$ is in an $X$-component, say $K$, of $G$. If $k \leq 2$, then the statement trivially holds. If $k \geq 3$, suppose, by contradiction, that $|V(p) \cap X|>1$. Let $w_{i}$ be the first vertex in $p$ not belonging to $V(K)$ (so that $w_{i} \in X$ ) and $w_{j}$ a vertex in $V(p) \cap X$ distinct from $w_{i}$ (so that $j>i$ ); one has that $w_{i-1} \in V(K) \cap N(X)$. Since $g(G)=m(G)$ and $X$ is a minimal vertex clique separator, by (2) in Theorem $11, X \cup\left\{w_{i-1}\right\}$ is a clique so that $w_{i-1}$ and $w_{j}$ are adjacent and, hence, $p$ is not an induced path (contradiction).

Lemma 13. Let $G$ be a graph such that $g(G)=m(G)$ and $X$ a subset of $V(G)$. If $Y$ is a minimal clique separator for two vertices in $X$, then $Y \subseteq I_{g}(X)$.

Proof. Let $u$ and $w$ be two vertices in $X$ such that $Y$ is a minimal clique separator for $u$ and $w$. Let $p=\left(u=w_{1}, \ldots, w_{i-1}, w_{i}, w_{i+1}, \ldots, w_{k}=w\right)$, $k \geq 3$, be a $u$-w geodesic. By Fact 2 and Lemma $12,|V(p) \cap Y|=1$. Let $V(p) \cap Y=\left\{w_{i}\right\}$. If $Y=\left\{w_{i}\right\}$ then the statement trivially holds. Otherwise, let $y$ be a vertex in $Y$ distinct from $w_{i}$ and let $K_{u}$ and $K_{w}$ be the $Y$ components of $G$ containing $u$ and $w$, respectively. Since $w_{i-1} \in V\left(K_{u}\right) \cap N(Y)$ and $w_{i+1} \in V\left(K_{w}\right) \cap N(Y)$, by (2) in Theorem 11, $y$ is adjacent to both $w_{i-1}$ and $w_{i+1}$. Therefore, $\left(w_{1}, \ldots, w_{i-1}, y, w_{i+1}, \ldots, w_{k}\right)$ is a $u$-w geodesic.

Lemma 14. Let $G$ be a graph such that $g(G)=m(G), X$ a minimal clique separator for two vertices $u_{1}$ and $u_{2}$, and $v$ a vertex in $X$. If $p_{1}$ is a $u_{1}-v$ geodesic and $p_{2}$ is a $v$ - $u_{2}$ geodesic, then $p_{1} p_{2}$ is a $u_{1}-u_{2}$ geodesic.

Proof. Suppose, by contradiction, that $p_{1} p_{2}$ is not a geodesic. Let $p$ be a $u_{1}-u_{2}$ geodesic. Let $v^{\prime}$ be a vertex in $X \cap V(p)$ and $p_{u_{1} v^{\prime}}$ and $p_{v^{\prime} u_{2}}$ the $u_{1}-v^{\prime}$ subpath and the $v^{\prime}-u_{2}$ subpath of $p$, respectively. Since $l(p)<l\left(p_{1} p_{2}\right)=l\left(p_{1}\right)+l\left(p_{2}\right)$, one has that $l\left(p_{u_{1} v^{\prime}}\right)<l\left(p_{1}\right)$ or $l\left(p_{v^{\prime} u_{2}}\right)<l\left(p_{2}\right)$. Assume, without loss of generality, that $l\left(p_{u_{1} v^{\prime}}\right)<l\left(p_{1}\right)$; let $K$ be the $X$-component of $G$ containing $u_{1}$ and $w$ the vertex preceding $v^{\prime}$ in $p_{u_{1} v^{\prime}}$. By Lemma $12, w \in V(K) \cap N(X)$, so that, by (2) in Theorem 11, $w$ is adjacent to $v$. Let $p_{u_{1}, w}$ be the $u_{1}-w$ subpath of $p$ and let $p^{\prime}=p_{u_{1}, w}(w, v)$. One has that $l\left(p^{\prime}\right)=l\left(p_{u_{1} v^{\prime}}\right)<l\left(p_{1}\right)$ (contradiction). 


\section{The joint of a vertex set in a prime component}

Let $G$ be a graph, $X$ a subset of $V(G)$ and $P$ a prime component of $G$. The joint of $X$ in $P$ is the set

$$
J(X, P)= \begin{cases}X, & \text { if } X \subseteq V(P), \\ X \cap V(P) \cup \bigcup_{K \in \mathcal{K}(X, P)} N(V(K)) \cap I_{g}(X), & \text { otherwise. }\end{cases}
$$

Fact 5. Let $G$ be a graph, $X$ a subset of $V(G)$ and $P$ a prime component of $G$. $J(X, P) \subseteq I_{g}(X) \cap V(P)$.

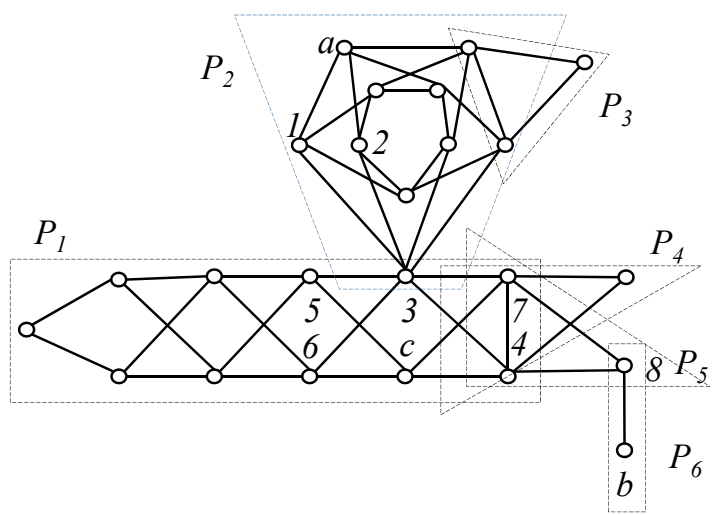

Figure 2:

Example 1. Consider the graph in Figure 2 and let $X=\{a, b, c\}$. One has $I_{g}(X)=\{a, b, c, 1,2, \ldots, 8\}, I_{g}(X) \cap V\left(P_{1}\right)=\{c, 3,4,5,6,7\}$, and $J\left(X, P_{1}\right)=$ $\{c, 3,4,7\}$.

In this section we will prove that, if $g(G)=m(G)$, then

$$
[X]_{g}=\bigcup_{P \in \mathcal{P}_{G}}[J(X, P)]_{g},
$$

Example 1 (continued). One has that $J\left(X, P_{1}\right)=\{c, 3,4,7\}, J\left(X, P_{2}\right)=$ $\{a, 3\}, J\left(X, P_{3}\right)=\emptyset, J\left(X, P_{4}\right)=\{4,7\}, J\left(X, P_{5}\right)=\{4,7,8\}$, and $J\left(X, P_{6}\right)=$ $\{b, 8\}$. Therefore, by Lemma 9 and Theorem 8, one has that $\left[J\left(X, P_{1}\right)\right]_{g}=$ $V\left(P_{1}\right),\left[J\left(X, P_{2}\right)\right]_{g}=V\left(P_{2}\right),\left[J\left(X, P_{3}\right)\right]_{g}=\emptyset,\left[J\left(X, P_{4}\right)\right]_{g}=\{4,7\},\left[J\left(X, P_{5}\right)\right]_{g}=$ $V\left(P_{5}\right)$, and $\left[J\left(X, P_{6}\right)\right]_{g}=V\left(P_{6}\right)$ so that $[X]_{g}=V\left(P_{1}\right) \cup V\left(P_{2}\right) \cup V\left(P_{5}\right) \cup V\left(P_{6}\right)$.

If we consider the graph in Figure 2 and the vertex set $X=\{a, b, c\}$, we can observe that $I_{g}\left(J\left(X, P_{1}\right)\right)=\{c, 3,4,5,6,7\}$, so that $I_{g}(X) \cap V\left(P_{1}\right)=$ 
$I_{g}\left(J\left(X, P_{1}\right)\right)$. We will prove now that the equality

$$
I_{g}(X) \cap V(P)=I_{g}(J(X, P))
$$

always holds.

Lemma 15. Let $G$ be a graph such that $g(G)=m(G), X$ a subset of $V(G)$, and $P$ a prime component of $G$. One has that $I_{g}(J(X, P))=I_{g}(X) \cap V(P)$.

Proof. Firstly, let us show that

$$
I_{g}(J(X, P)) \subseteq I_{g}(X) \cap V(P) .
$$

By Fact $5, J(X, P) \subseteq V(P)$ and, hence, by Theorem 8 and 3 in Fact 1 , $I_{g}(J(X, P)) \subseteq V(P)$. Therefore, in order to show that $I_{g}(J(X, P)) \subseteq I_{g}(X) \cap$ $V(P)$ it is sufficient to show that $I_{g}(J(X, P)) \subseteq I_{g}(X)$. Let $v$ be a vertex in $I_{g}(J(X, P))$. Let $p$ be a geodesic between two (not necessarily distinct) vertices $u$ and $w$ belonging to $J(X, P)$ such that $v \in V(p)$. Distinguish two cases.

Case 1. There exists $K \in \mathcal{K}(X, P)$ such that both $u$ and $w$ are in $N(V(K))$. By Lemma $5, N(V(K))$ is a clique and, hence, either $u=w$ or $u$ and $w$ are adjacent; therefore, either $v \in V(p)=\{u\}$ or $v \in V(p)=\{u, w\}$. Since, by Fact 5 , both $u$ and $w$ are in $I_{g}(X)$, it follows that $v \in I_{g}(X)$.

Case 2. There is no $K \in \mathcal{K}(X, P)$ such that both $u$ and $w$ are in $N(V(K))$. If both $u$ and $w$ are in $X$, then, trivially, $v \in I_{g}(X)$. Otherwise, assume without loss of generality, that $u \notin X$. By the definition of joint of $X$ in $P$, there exists a connected component $K_{u} \in \mathcal{K}(X, P)$ such that $u \in N\left(V\left(K_{u}\right)\right.$ ) (so that $\left.w \notin N\left(V\left(K_{u}\right)\right)\right)$. Let $u^{\prime}$ be a vertex in $X \cap V\left(K_{u}\right)$ and let $p_{u^{\prime} u}$ be a $u^{\prime}$-u geodesic. Since, by 2 in Theorem $7, N\left(V\left(K_{u}\right)\right)$ is a minimal clique separator for $u^{\prime}$ and $w$, by Lemma 14 , the $u^{\prime}-w$ path $p^{\prime}=p_{u^{\prime} u} p$ is a geodesic. Therefore, if $w \in X$, then, since $v \in V(p) \subseteq V\left(p^{\prime}\right), v \in I_{g}(X)$. Otherwise, by the definition of joint of $X$ in $P$, there exists a connected component $K_{w} \in \mathcal{K}(X, P)$ distinct from $K_{u}$ such that $w \in N\left(V\left(K_{w}\right)\right)$. Let $w^{\prime}$ be a vertex in $X \cap V\left(K_{w}\right)$ and let $p_{w w^{\prime}}$ be a $w-w^{\prime}$ geodesic. Since $u \in V(P) \backslash N\left(V\left(K_{w}\right)\right)$, by 2 in Theorem 7, $N\left(V\left(K_{w}\right)\right)$ is a minimal clique separator for $u$ and $w^{\prime}$. By 1 in Theorem 7, there exists an $N\left(V\left(K_{w}\right)\right)$-component $K^{\prime}$ of $G$ containing $u$. Since, by Lemma $12, u$ is the unique vertex in $V\left(p_{u^{\prime} u}\right) \cap N\left(V\left(K_{u}\right)\right)$ (and, hence, is the unique vertex in $\left.V\left(p_{u^{\prime} u}\right) \cap V(P)\right)$ and $u \notin N\left(V\left(K_{w}\right)\right)$, one has that $u^{\prime} \in V\left(K^{\prime}\right)$ so that, by 2 in Theorem 7, $N\left(V\left(K_{w}\right)\right)$ is a minimal clique separator for $u^{\prime}$ and $w^{\prime}$. Therefore, by Lemma 14 , the $u^{\prime}-w^{\prime}$ path $p^{\prime \prime}=p^{\prime} p_{w w^{\prime}}$ is a $u^{\prime}-w^{\prime}$ geodesic. Since $v \in V(p) \subseteq V\left(p^{\prime \prime}\right)$, one has that $v \in I_{g}(X)$.

Now let us show that:

$$
I_{g}(J(X, P)) \supseteq I_{g}(X) \cap V(P) .
$$

Let $v$ be a vertex in $I_{g}(X) \cap V(P)$. Let $u$ and $w$ be two vertices in $X$ such that $v$ is on a $u$ - $w$ geodesic $p=\left(u=v_{1}, v_{2}, \ldots, v_{h}=w\right), h \geq 1$, and let $v=v_{i}$, $1 \leq i \leq h$. Let $r=\min \left(l \mid v_{l} \in V(P)\right)$ and $s=\max \left(l \mid v_{l} \in V(P)\right)$. Let us show that: 
(a) $v_{r}$ is in $J(X, P)$.

If $r=1$ then (a) trivially holds. If $r>1$, then $u \notin V(P)$. Let $K$ be the connected component in $\mathcal{K}(X, P)$ such that $u \in V(K)$. Since, $v_{r} \in N(V(K))$ and $v_{r} \in V(p) \subseteq I_{g}(X)$, (a) holds. Analogously, it is possible show that:

(b) $v_{s}$ is in $J(X, P)$.

From $(a)$ and $(b)$, it follows that $v \in I_{g}(J(X, P))$.

Lemma 16. Let $G$ be a graph such that $g(G)=m(G), X$ a subset of $V(G)$, and $P$ a prime component of $G$. For every $j, j \geq 1$,

$$
I_{g}^{j-1}\left(I_{g}(X) \cap V(P)\right)=I_{g}^{j}(X) \cap V(P) .
$$

Proof. Since $I_{g}(X) \cap V(P) \subseteq I_{g}(X)$, for every $j, j \geq 1$,

$$
I_{g}^{j-1}\left(I_{g}(X) \cap V(P)\right) \subseteq I_{g}^{j-1}\left(I_{g}(X)\right)=I_{g}^{j}(X)
$$

and, since $I_{g}(X) \cap V(P) \subseteq V(P)$, by Theorem 8 and 3 in Fact 1 , for every $j$, $j \geq 1$,

$$
I_{g}^{j-1}\left(I_{g}(X) \cap V(P)\right) \subseteq V(P) .
$$

Therefore, for every $j, j \geq 1$,

$$
I_{g}^{j-1}\left(I_{g}(X) \cap V(P)\right) \subseteq I_{g}^{j}(X) \cap V(P) .
$$

Now, let us prove, by induction, that, for every $j, j \geq 1$,

$$
I_{g}^{j}(X) \cap V(P) \subseteq I_{g}^{j-1}\left(I_{g}(X) \cap V(P)\right) .
$$

Basis. $j=1$. Trivial.

Induction. $j>1$. Let $v \in I_{g}^{j}(X) \cap V(P)$. If $v \in I_{g}^{j-1}(X)$, then, by inductive hypothesis, $v \in I_{g}^{j-2}\left(I_{g}(X) \cap V(P)\right) \subseteq I_{g}^{j-1}\left(I_{g}(X) \cap V(P)\right)$. Therefore assume that $v \in I_{g}^{j}(X) \backslash I_{g}^{j-1}(X)$. Observe that, since $v \notin J(X, P)$ (otherwise, by Fact $5, v$ would be in $\left.I_{g}(X) \subseteq I_{g}^{j-1}(X)\right), v \notin V(\widehat{H})$, for every $H \in \mathcal{K}(X, P)$ and, hence, by Lemma 10,

(a) $v \notin V(\widehat{H})$, for every $H \in \mathcal{K}\left(I_{g}^{j-1}(X), P\right)$.

Let $u$ and $w$ be two vertices in $I_{g}^{j-1}(X)$ such that $v$ is on a $u$ - $w$ geodesic $p=(u=$ $\left.v_{1}, v_{2}, \ldots, v_{h}=w\right), h \geq 3$, and let $v=v_{i}, 1<i<h$. Let $r=\min \left(l \mid v_{l} \in V(P)\right)$ and $s=\max \left(l \mid v_{l} \in V(P)\right)$. Let us show that:

(b) $v_{r} \in I_{g}^{j-1}(X)$.

If $r=1$, then $(b)$ trivially holds. If $r>1$, then $u \notin V(P)$. Let $K_{u}$ be the connected component of $G-V(P)$ containing $u$. Observe that, by $(a)$, $v \in V(P) \backslash N\left(V\left(K_{u}\right)\right)$; therefore, by 2 in Theorem $7, N\left(V\left(K_{u}\right)\right)$ is a minimal clique separator for $u$ and $v$ and, hence, by Lemma $2, u$ and $v$ are in two distinct $N\left(V\left(K_{u}\right)\right)$-components of $G$. Let $K$ be the $N\left(V\left(K_{u}\right)\right)$-component of 
$G$ containing $v$. Since $v$ is on a $u$ - $w$ geodesic and $u \notin V(K)$, by Lemma 3 , one has that $w \in V(K)$. It follows (since $w \in I_{g}^{j-1}(X)$ ) that $I_{g}^{j-1}(X) \cap V(K) \neq \emptyset$ and, hence, by Corollary $4, X \cap V(K) \neq \emptyset$. On the other hand, by Lemma 10, $X \cap V\left(K_{u}\right) \neq \emptyset$. Therefore, by 2 in Theorem $7, N\left(V\left(K_{u}\right)\right)$ is a minimal clique separator for two vertices in $X$ and, hence, by Lemma 13, $N\left(V\left(K_{u}\right)\right) \subseteq I_{g}(X)$. Since, $v_{r} \in N\left(V\left(K_{u}\right)\right),(b)$ is proved. Analogously, it is possible show that

(c) $v_{s} \in I_{g}^{j-1}(X)$.

Consider the $v_{r}-v_{s}$ subpath $p^{\prime}$ of $p$. One has that $p^{\prime}$ is a $v_{r}-v_{s}$ geodesic and $v \in V\left(p^{\prime}\right)$. Since, by $(b)$ and $(c)$, both $v_{r}$ and $v_{s}$ are in $I_{g}^{j-1}(X) \cap V(P)$, by inductive hypothesis, both $v_{r}$ and $v_{s}$ are in $I_{g}^{j-2}\left(I_{g}(X) \cap V(P)\right)$ and, hence, $v \in I_{g}^{j-1}\left(I_{g}(X) \cap V(P)\right)$.

Theorem 17. Let $G$ be a graph such that $g(G)=m(G)$ and $X$ a subset of $V(G)$. One has that

$$
[X]_{g}=\bigcup_{P \in \mathcal{P}_{G}}[J(X, P)]_{g},
$$

Proof. Firstly, let us show that, for every prime component $P$ of $G$ :

$$
[J(X, P)]_{g} \subseteq[X]_{g} \cap V(P) .
$$

By 2 in Fact 1 , for every prime component $P$ of $G$, one has that

$$
[J(X, P)]_{g} \subseteq\left[I_{g}(J(X, P))\right]_{g} .
$$

Furthermore, by Theorem 8 and 3 in Fact 1, for every prime component $P$ of $G$, one has that

$$
\left[I_{g}(X) \cap V(P)\right]_{g} \subseteq V(P) .
$$

Finally, since, for every prime component $P$ of $G, I_{g}(X) \cap V(P) \subseteq I_{g}(X)$ and, by 2 in Fact $1,\left[I_{g}(X)\right]_{g}=[X]_{g}$, one has that, for every prime component $P$ of $G$,

$$
\left[I_{g}(X) \cap V(P)\right]_{g} \subseteq[X]_{g} .
$$

Therefore, (a) follows from (a.1), (a.2), (a.3), and Lemma 15.

Let us show now that:

$$
[X]_{g} \subseteq \bigcup_{V(P) \in \mathcal{P}_{G}}[J(X, P)]_{g}
$$

Let $h=\operatorname{gin}(X)$. If $h=0, X=[X]_{g}$ and, hence, since $X \cap V(P) \subseteq J(X, P)$, one has that $[X]_{g} \cap V(P) \subseteq[J(X, P)]_{g}$; therefore (b) follows fron Fact 4. If $h \geq 1$, then one has 


$$
\begin{aligned}
{[X]_{g} } & =\bigcup_{V(P) \in \mathcal{P}_{G}}[X]_{g} \cap V(P)= & & \text { (Fact 4) } \\
& =\bigcup_{V(P) \in \mathcal{P}_{G}} I_{g}^{h}(X) \cap V(P)= & & (1 \text { in Fact 1) } \\
& =\bigcup_{V(P) \in \mathcal{P}_{G}} I_{g}^{h-1}\left(I_{g}(X) \cap V(P)\right) \subseteq & & \text { (Lemma 16) } \\
& \subseteq \bigcup_{V(P) \in \mathcal{P}_{G}}\left[I_{g}(X) \cap V(P)\right]_{g}= & & (1 \text { in Fact 1) } \\
& =\bigcup_{V(P) \in \mathcal{P}_{G}}\left[I_{g}(J(X, P))\right]_{g}= & & \text { (Lemma 15) } \\
& =\bigcup_{V(P) \in \mathcal{P}_{G}}[J(X, P)]_{g} & & \text { (2 in Fact 1) }
\end{aligned}
$$

\section{The geodetic iteration number}

In this section we state the results concerning the geodetic iteration number of a set of vertices of a graph in the class $\Gamma$ (Theorem 18) and the geodetic iteration number of a graph in the class $\Gamma$ (see Theorems 19 and 20).

Theorem 18. Let $G$ be a graph such that $g(G)=m(G)$ and $X$ a subset of $V(G)$. One has that:

1. $\operatorname{gin}(X)=0$, if $X$ is g-convex;

2. $\operatorname{gin}(X)=1$, if $X$ is not $g$-convex and, for every prime component $P$ of $G, I_{g}(X) \cap V(P)$ is g-convex;

3. $\operatorname{gin}(X)=\max \left\{\operatorname{gin}(J(X, P)) \mid V(P) \in \mathcal{P}_{G}\right\}$, otherwise.

Proof. Proof of 1. Trivial.

Proof of 2. Let $h=\operatorname{gin}(X)$; since $X$ is not $g$-convex, $h \geq 1$. One has that 


$$
\begin{array}{rlr}
{\left[I_{g}(X)\right]_{g}} & =[X]_{g}= & \text { (2 in Fact 1) } \\
& =I_{g}^{h}(X)= & \text { (1 in Fact 1) } \\
& =\bigcup_{V(P) \in \mathcal{P}_{G}} I_{g}^{h}(X) \cap V(P)= & \text { (Fact 4) } \\
& =\bigcup_{V(P) \in \mathcal{P}_{G}} I_{g}^{h-1}\left(I_{g}(X) \cap V(P)\right)= & \text { (Lemma 16) } \\
& =\bigcup_{V(P) \in \mathcal{P}_{G}} I_{g}(X) \cap V(P)= & \left(I_{g}(X) \cap V(P) \text { is } g\right. \text {-convex) } \\
& =I_{g}(X)
\end{array}
$$

so that $I_{g}(X)$ is $g$-convex and, hence, $\operatorname{gin}(X)=1$

Proof of 3. Let $h=\operatorname{gin}(X)$ and $k=\max \left\{\operatorname{gin}(J(X, P)) \mid V(P) \in \mathcal{P}_{G}\right\}$. Since $X$ is not $g$-convex, $h \geq 1$; furthermore, $k \geq 1$ (otherwise, for every prime component $P$ of $G, J(X, P)$ would be $g$-convex and, hence, by Lemma 15 , $I_{g}(X) \cap V(P)$ would be $g$-convex). Suppose, by contradiction, that $h \neq k$. If $h>k$, then one has that

$$
\begin{array}{rlr}
\bigcup_{V(P) \in \mathcal{P}_{G}}[J(X, P)]_{g} & =\bigcup_{V(P) \in \mathcal{P}_{G}} I_{g}^{k}(J(X, P))= & (1 \text { in Fact 1) } \\
& =\bigcup_{V(P) \in \mathcal{P}_{G}} I_{g}^{k-1}\left(I_{g}(X) \cap V(P)\right)= & (\text { Lemma 15) } \\
& =\bigcup_{V(P) \in \mathcal{P}_{G}} I_{g}^{k}(X) \cap V(P) \subsetneq \\
& \subsetneq \bigcup_{V(P) \in \mathcal{P}_{G}} I_{g}^{h}(X) \cap V(P)= \\
& =\bigcup_{V(P) \in \mathcal{P}_{G}}[X]_{g} \cap V(P)= & (1 \text { in Fact 1) } \\
& =[X]_{g}
\end{array}
$$

If $h<k$, then, one has that 


$$
\begin{array}{rlrl}
{[X]_{g}} & =\bigcup_{V(P) \in \mathcal{P}_{G}}[X]_{g} \cap V(P)= & \text { (Fact 4) } \\
& =\bigcup_{V(P) \in \mathcal{P}_{G}} I_{g}^{h}(X) \cap V(P)= & & \text { (1 in Fact 1) } \\
& =\bigcup_{V(P) \in \mathcal{P}_{G}} I_{g}^{h-1}\left(I_{g}(X) \cap V(P)\right)= & & \text { (Lemma 16) } \\
& =\bigcup_{V(P) \in \mathcal{P}_{G}} I_{g}^{h}(J(X, P)) \subsetneq & & \text { (Lemma 15) } \\
& \subsetneq \bigcup_{V(P) \in \mathcal{P}_{G}} I_{g}^{k}(J(X, P))=\bigcup_{V(P) \in \mathcal{P}_{G}}[J(X, P)]_{g} & & \text { (1 in Fact 1) }
\end{array}
$$

In both cases, by Theorem 17, a contradiction arises.

Theorem 19. Let $G$ be a graph such that $g(G)=m(G)$. One has that:

1. $\operatorname{gin}(G)=0$, if $G$ is complete;

2. $\operatorname{gin}(G)=1$, if $G$ is not complete and is a block grah;

3. $\operatorname{gin}(G)=\max \left\{\operatorname{gin}(P) \mid V(P) \in \mathcal{P}_{G}\right\}$, otherwise.

Proof. Proof of 1. If $G$ is complete, then every subset $X$ of $V(G)$ either is empty or is a clique and, hence, is $g$-convex; therefore, $\operatorname{gin}(G)=0$.

Proof of 2. Let $X$ be a subset of $V(G)$. Since $G$ is a block graph, for every prime component $P$ of $G, I_{g}(X) \cap V(P)$ is empty or is a clique and, hence, is $g$-convex. Therefore, by 1 and 2 in Theorem $18, \operatorname{gin}(X) \leq 1$. It follows that $\operatorname{gin}(G) \leq 1$. Since $G$ is not complete, $G$ has at least two nonadjacent vertices $u$ and $v$; since $\{u, v\}$ is not $g$-convex $\operatorname{gin}(G)=1$.

Proof of 3. Let $k=\max \left\{\operatorname{gin}(P) \mid V(P) \in \mathcal{P}_{G}\right\}$. Since $G$ is not a block graph, there exists a prime component of $G$ containing two nonadjacent vertices $u$ and $v$; since $\operatorname{gin}(\{u, v\}) \geq 1$, one has that $k \geq 1$. Let $X$ be a subset of $V(G)$. If $X$ is $g$-convex or, for every prime component $P$ of $G, I_{g}(X) \cap V(P)$ is $g$-convex, then by 1 and 2 in Theorem $18, \operatorname{gin}(X) \leq k$. Otherwise, by 3 in Theorem 18 ,

$$
\operatorname{gin}(X)=\max \left\{\operatorname{gin}(J(X, P)) \mid V(P) \in \mathcal{P}_{G}\right\} \leq k .
$$

Therefore, $\operatorname{gin}(G) \leq k$. Let $P^{\prime}$ be a prime component of $G$ such that $\operatorname{gin}\left(P^{\prime}\right)=k$ and $X^{\prime}$ a subset of $V(P)$ such that $\operatorname{gin}\left(X^{\prime}\right)=\operatorname{gin}\left(P^{\prime}\right)$. Then $\operatorname{gin}\left(X^{\prime}\right)=k$ and, hence, $\operatorname{gin}(G)=k$.

Example 1 (continued). Let $G$ be the graph in Figure 2. One has that $\operatorname{gin}\left(P_{1}\right)=4, \operatorname{gin}\left(P_{2}\right)=3, \operatorname{gin}\left(P_{3}\right)=\operatorname{gin}\left(P_{4}\right)=\operatorname{gin}\left(P_{5}\right)=\operatorname{gin}\left(P_{6}\right)=0$, and, hence, $\operatorname{gin}(G)=\operatorname{gin}\left(P_{1}\right)=4$. 
Theorem 20. Let $G$ be a prime graph such that $g(G)=m(G)$. One has that:

1. $\operatorname{gin}(G)=0$, if $G$ is complete;

2. $\operatorname{gin}(G)=\max \{\operatorname{gin}(\{u, v\}) \mid u v \notin E(G)\}$, otherwise.

Proof. Proof of 1. Trivial.

Proof of 2. Let $h=\operatorname{gin}(G)$ and $k=\max \{\operatorname{gin}(\{u, v\}) \mid u v \notin E(G)\}$; observe that $h \geq k \geq 1$. Suppose, by contradiction, that $h>k$. Let $X$ be a subset of $V(G)$ such that $\operatorname{gin}(X)=h$. Since $h \geq 1, X$ cannot be empty and cannot be a clique. Let $x$ and $y$ be two nonadjacent vertices in $X$. Since $g(G)=m(G)$, by Lemma 9, one has that:

$$
V(G)=[\{x, y\}]_{g}=I_{g}^{k}(\{x, y\}) \subseteq I_{g}^{k}(X) \subsetneq I_{g}^{h}(X)=[X]_{g}=V(G)
$$

which is a contradiction.

\section{Computing the geodetic iteration number}

By Theorems 19 and 20, in order to compute the geodetic iteration number of a graph $G \in \Gamma$ that is neither complete nor a block graph we have to compute the prime components of $G$ and then to compute $\operatorname{gin}(\{u, v\})$, for every pair of nonadjacent vertices $u$ and $v$ that are both in a prime component of $G$. The prime components of a graph $G$ can be computed using the $O(n m)$ decomposition algorithm given in [7] and modified by [8]. Furthermore, we can compute $\operatorname{gin}(\{u, v\})$ in $O(n m)$ by applying the algorithm in Figure 3 which is substantially the process described in [9] to compute the $g$-convex hull of a set of vertices. Therefore, the following holds.

input: a graph $G$ and a subset $X$ of $V(G)$;

output: $\operatorname{gin}(X)$;

begin

$\operatorname{gin}(X):=0$

$I^{0}:=X$

for every $u \in I^{0}$ do $I^{1}:=\bigcup_{v \in I^{0}} I_{g}(u, v)$;

while $I^{0} \neq I^{1}$ do

begin

$\operatorname{gin}(X):=\operatorname{gin}(X)+1$

for every $u \in I^{1} \backslash I^{0}$ do $I^{2}:=\bigcup_{v \in I^{1}} I_{g}(u, v)$;

$I^{0}:=I^{1}$;

$I^{1}:=I^{2}$

end

end.

Figure 3: 
Theorem 21. Let $G$ be a graph such that $g(G)=m(G)$. The geodetic iteration number of $G$ can be computed in $O\left(n^{3} m\right)$.

Let us discuss now the complexity of computing the geodetic iteration number of a bipartite distance-hereditary graph. In [10] it is proved that the class of bipartite distance-hereditary graphs is properly contained in a proper sublass, say $\Gamma^{\prime}$ (in [10], such a class is the class of cross-cyclic graphs having no cycle of length 3), of $\Gamma$ and that for a graph in $\Gamma^{\prime}$ the $g$-convex hull of a set of vertices $X$ coincides with the $2 g$-convex hull of $I_{g}(X)$, where the $2 g$-convex hull of a vertex set is defined analogously to $g$-convex hull with the difference that only geodesics of length 2 are considered. Therefore, if $G$ is a bipartite distance-hereditary graph, in order to compute $\operatorname{gin}(\{u, v\})$ we can use the $O(m)$ algorithm in Figure 4 which is substantially the algorithm provided in [10] to compute the $g$-convex hull of a set of vertices in a graph belonging to $\Gamma^{\prime}$. This algorithm firstly computes $I_{g}(X)$, and then add a vertex $v$ to $I_{g}^{j}(X), j \geq 2$, if $v$ is adjacent to at least two vertices in $I_{g}^{j-1}(X)$ and, hence, is on a geodesic of lenght 2 between two vertices in $I_{g}^{j-1}(X)$. Therefore the following holds.

input: a bipartite distance-hereditary graph $G$ and a subset $X$ of $V(G)$;

output: $\operatorname{gin}(X)$;

begin

$I^{0}:=X$

$\operatorname{gin}(X):=0$

$I^{1}:=I_{g}(X)$;

$I^{2}:=I^{1}$

for every $v \in V(G) \backslash X$ do $\operatorname{adj}(v):=0$;

while $I^{0} \neq I^{1}$ do

begin

$\operatorname{gin}(X):=\operatorname{gin}(X)+1$

for every $u \in I^{1} \backslash I^{0}$ do

for every $v \in N(u) \backslash I^{1}$ do

begin

$\operatorname{adj}(v):=\operatorname{adj}(v)+1$;

if $\operatorname{adj}(v)=2$ then $I^{2}:=I^{2} \cup\{v\}$

end

$I^{0}:=I^{1}$

$I^{1}:=I^{2}$

end

end.

Figure 4:

Theorem 22. The geodetic iteration number of a bipartite distance-hereditary graph can be computed in $O\left(n^{2} m\right)$ time. 


\section{References}

[1] M. C. Dourado, R. A. de Oliveira, F. Protti, D. Rautenbach, On the geodetic iteration number of distance-hereditary graphs, Discrete Mathematics 339 (2) (2016) 489-498. doi:10.1016/j.disc.2015.09.025.

URL http://dx.doi.org/10.1016/j.disc.2015.09.025

[2] M. Farber, R. E. Jamison, Convexity in graphs and hypergraphs, SIAM J. of Algebraic Discrete Mathematics 7 (3) (1986) 433-444.

[3] V. P. Soltan, Metric convexity in graphs, Studia Univ. Babes-Bolyai Math. 36 (4) (1991) 3-43.

[4] P. Duchet, Convex sets in graphs II: minimal path convexity, J. Combinatorial Theory Series B 44 (3) (1988) 307-316.

[5] F. M. Malvestuto, M. Mezzini, M. Moscarini, Characteristic properties and recognition of graphs in which geodesic and monophonic convexities are equivalent, Discrete Math., Alg. and Appl. 4 (4) (2012) 1-14.

[6] F. Harary, J. Nieminen, Convexity in graphs, J. Differential Geometry 16 (1981) 185-190.

[7] R. E. Tarjan, Decomposition by clique separators, Discrete Mathematics 55 (2) (1985) 221-232.

[8] H.-G. Leimer, Optimal decomposition by clique separators, Discrete Mathematics 113 (1-3) (1993) 99-123.

[9] M. C. Dourado, J. G. Gimbel, J. Kratochvíl, F. Protti, J. L. Szwarcfiter, On the computation of the hull number of a graph, Discrete Mathematics 309 (18) (2009) 5668-5674. doi:10.1016/j.disc.2008.04.020.

URL http://dx.doi.org/10.1016/j.disc.2008.04.020

[10] M. Moscarini, F. M. Malvestuto, Two classes of graphs in which some problems related to convexity are efficiently solvable, Discrete Math., Alg. and Appl. 10 (3) (2018) 1-22. doi:10.1142/S1793830918500428.

URL https://doi.org/10.1142/S1793830918500428 